NASA Contractor Report 3086

\title{
Attenuation of Sound in a Low Mach Number Nozzle Flow
}

M. S. Howe

CONTRACT NAS1-14611-12

FEBRUARY 1979 


\section{NASA Contractor Report 3086}

\section{Attenuation of Sound in a Low Mach Number Nozzle Flow}

M. S. Howe

Bolt Beranek and Newman Inc.

Cambridge, Massachusetts

Prepared for

Langley Research Center

under Contract NAS1-14611-12

\section{N/Sก}

National Aeronautics and Space Administration

Scientific and Technical Information Office 
TABLE OF CONTENTS

page

LIST OF FIGURES $\ldots \ldots \ldots \ldots \ldots \ldots \ldots \ldots \ldots \ldots \ldots \ldots \ldots \ldots$

SUMMARY $\ldots \ldots \ldots \ldots \ldots \ldots \ldots \ldots \ldots \ldots \ldots \ldots \ldots \ldots \ldots \ldots \ldots \ldots \ldots \ldots \ldots$

1. INTRODUCTION $\ldots \ldots \ldots \ldots \ldots \ldots \ldots \ldots \ldots \ldots \ldots \ldots \ldots \ldots \ldots \ldots$

2. THE RADIATION OF INTERNALLY GENERATED SOUND FROM

A LOW MACH NUMBER NOZZLE FLOW ............. 5

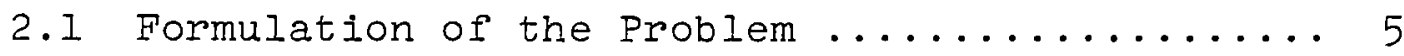

2.2 Energy Flux Within the Jet Pipe .......... 8

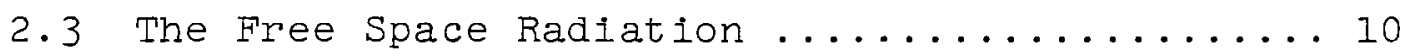

3. THE FLUX OF ENERGY THROUGH THE NOZZLE .......... 13

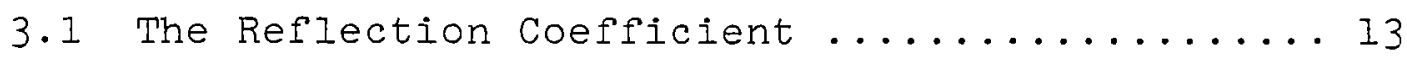

3.2 The Mechanism of Hydrodynamic Attenuation ..... 17

3.3 Exterior Flow Models ................. 21

4. THE RADIATED SOUND POWER: COMPARISON

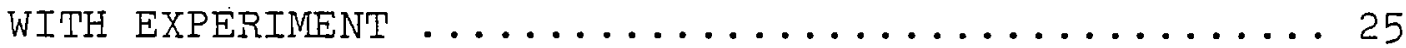

5. CONCLUSION $\ldots \ldots \ldots \ldots \ldots \ldots \ldots \ldots \ldots \ldots \ldots \ldots \ldots \ldots \ldots \ldots \ldots \ldots$

APPENDIX: Compact Green's Function for an

Axi-Symmetric Nozzle ............... 33

Incompressible Pulsatile Nozzle Flow ...... 35

The Case of a Finite Width Shear Layer .... 38

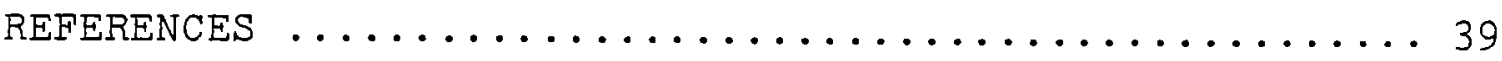




\section{LIST OF FIGURES}

page

Figure 1. Schematic illustration of the configuration considered in the analysis of the emission of low frequency sound from a jet pipe in the presence of a mean nozzle flow .......... 6

2. Predicted field shape characteristics for $\mathrm{ka}=0.24$ and $\mathrm{M}_{\mathrm{J}}=0.3$; Case I vortex sheet model; Case II --.---, fintte width shear layer model; angle $\theta$ is measured from the downstream direction of the jet axis, and the experimental points are taken from Pinker and Bryce $(1976): \Delta-\mathrm{ka}=0.24 ; 0-\mathrm{ka}=0.6$ at $\mathrm{M}_{\mathrm{J}}=0.327$

3. Measured ratio of the far field sound power $W_{F}$ to the nozzle power flux $W_{T}$ as a function of the nozzle exit Helmholtz number ka for various values of the jet Mach number $\mathrm{M}_{J}$ (Bechert,

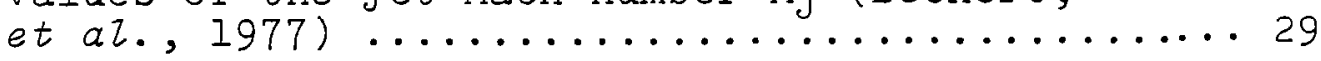

4. Comparison of predicted and measured ratio $\mathrm{W}_{\mathrm{F}} / \mathrm{W}_{\mathrm{T}}$ as a function of $\mathrm{ka}$ for $\mathrm{M}_{\mathrm{J}}=0.3$.

Experiment: 000 (Bechert, et al., 1977); Theory: Case I, vortex sheet model, -----Case II, finite width shear layer

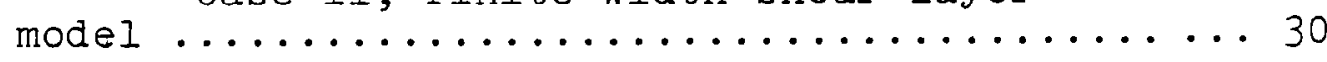




\section{SUMMARY}

This report examines the energy conversion mechanisms which govern the emission of low frequency sound from an axisymmetric jet pipe of arbitrary nozzle contraction ratio in the case of low Mach number nozzle flow. The incident acoustic energy which escapes from the nozzle is partitioned between two distinct disturbances in the exterior fluid. The first of these is the free space radiation, whose directivity is equivalent to that produced by monopole and dipole sources. Second, essentially incompressible vortex waves are excited by the shedding of vorticity from the nozzle lip, and may be associated with the large scale instabilities of the jet. Two linearized theoretical models are discussed. One of these is an exact linear theory in which the boundary of the jet is treated as an unstable vortex sheet. The second assumes that the finite width of the mean shear layer of the real jet cannot be neglected. The analytical results are shown to compare favorably with recent attenuation measurements. 


\section{INTRODUCTION}

This report examines the energy conversion mechanisms involved in the emission of sound from the interior of a jet pipe in the presence of a subsonic nozzle flow. This is particularly relevant to the problem of "excess" or "core" noise produced by unsteady combustion and turbine blading in the jet pipe of an aeroengine. It is also of interest in connection with the energy balance associated with the generation of resonant oscillations in the pipe and in musical instruments such as the flute.

According to experiments of Crow (1972) and of Gerend, Kumasaki and Roundhill (1973), upstream generated sound is significantly amplified by passage through the jet at subsonic velocities, the additional radiation being attributed to the excitation of instability waves of the jet. This conclusion has been challenged by Moore (1977) and by Bechert, Michel and Pfizenmaier (1977) who pointed out that it was based on measurements of the acoustic intensity at a single far field location. In a series of carefully conducted experiments Moore demonstrated that over a wide frequency range and for jet Mach numbers lying between 0.1 - 0.9, there is no significant overall radiation from the instability mode at the excitation frequency.

This question was investigated by Bechert, Michel and Pfizenmaier (1977) using an acoustic tone generated within the jet pipe by means of a system of matched loudspeakers. Although their experiment was confined to the case of a cold subsonic jet, which precluded a strict comparison with the earlier work, the absence of amplification at the tonal frequency was confirmed. Moreover, at sufficiently low acoustic frequencies, specifically for Helmholtz numbers ka less than unity $-k$ being the acoustic wavenumber and a the nozzle exit radius - a considerable attenuation of the tone was observed during its emission through the nozzle flow into free space, an effect also reported by Moore (1977), and amounted to $15 \mathrm{~dB}$ or more for ka 0.2. A high level of tonal excitation is known to bring about an overall increase in the broadband noise produced by the jet [Bechert and Pfizenmaier (1975a), Moore (1977)], but Bechert et al. (1977) were able to show that this additional radiation in no way compensates for the strong attenuation of the tone, the relationship between the broadband amplification and the excitation amplitude being essentially nonlinear. 
Munt (1977) has described in detail a linearized analytical theory of the radiation of sound from a circular cylindrical pipe in the presence of a subsonic nozzle flow. The jet shear layer was approximated by an infinitely thin cylindrical vortex sheet, and free space radiation directivities calculated from this model were shown to be in excellent agreement with field shape data obtained by Pinker and Bryce (1976) using a jet pipe with a conical nozzle. This led Bechert, Michel and Pfizenmaier (1977) to suggest that the same theory could well account for the attenuation observed in their experiment at low frequencies. In this report we shall verify that this is indeed the case. No direct use will be made of Munt's formulae, however, since, although valid over a wide range of conditions, they offer no insight into the nature of the physical mechanisms which are called into play during the passage of an acoustic disturbance through the nozzle.

The interaction of an acoustic tone with low Mach number nozzle flow has been studied in relation to laminar-turbulent transition in a separated boundary layer. Brown (1935) and the experiments of Freymuth (1966) indicate that the influence of the sound on the free shear layer of the jet is restricted to the region close to the nozzle lip. Bechert and Pfizenmaier (1975b) examined the nature of the flow near the lip, and concluded that at sufficiently small strouhal numbers based on boundary layer width, the disturbed flow leaves the trailing edge tangentially, in accordance with the KuttaJoukowski hypothesis. We shall argue below that an attenuation of the acoustic field is necessary in order to energize the essentially incompressible, unsteady flow associated with the vorticity that must be shed from the lip to satisfy the Kutta condition. This may involve the growth of spatial instabilities of the jet, and in this case the attenuation may be regarded as being necessary to maintain the corresponding large scale 'coherent structures'. Of course, shed vorticity and instability waves are known to produce sound by their subsequent interaction with the nozzle, but at low frequencies the radiated sound power is of order $\mathrm{M}_{\mathrm{J}}(\mathrm{ka})_{2}$ relative to the power loss from the incident sound wave, $\mathrm{M}_{J}$ being the Mach number of the jet. This is accordingly a situation in which the production of aerodynamic quadrupole sources (Lighthill 1952), in the form of initially organized vortical disturbances, results in an overall reduction in the acoustic energy!

All available theories of jet-acoustic interaction (e.g., Crighton 1972; Savkar 1975; Munt 1977) employ a vortex sheet representation of the free shear layer and impose the Kutta condition. The Strouhal numbers of interest in the present discussion are sufficiently small to justify the application 
of this condition. However, the experiments of Pinker and Bryce (1976) and the results reported by Savkar (1975) indicate that there is no significant excitation of the instability mode for a cold jet operating at low subsonic Mach numbers. This suggests that it may be necessary to take account of the finite width of the mean shear layer, and indeed it may be argued that Pinker and Bryce's experimental results reveal that close to the nozzle lip, the radial length scale of the unsteady shed vorticity is much smaller than that of the shear layer.

In this report the attenuation of the sound will be discussed in terms of Lighthill's (1952) acoustic analogy theory of aerodynamic sound by means of the formulation proposed by the author (Howe 1975). It will be assumed that the acoustic wavelength is large compared with the radius of the jet pipe, and this will enable the analysis to take account of an arbitrary contraction in the cross-sectional area of the pipe at the nozzle. The general problem is formulated in Sec. 2 and the characteristics of the free space radiation field are deduced. The mechanism of energy transfer to the essentially incompressible vortex motions of the jet is described in Sec. 3; specific details are given for an exterior shear flow modelled by a vortex sheet, and also for an approximate treatment of the case of finite shear layer width (Sec. 4). The predictions of the analysis are discussed in relation to the experiments of Pinker \& Bryce (1976) and Bechert, Michel \& Pfizenmaier (1977). Various analytical results are collected together in an appendix. 


\section{THE RADIATION OF INTERNALLY GENERATED SOUND FROM A LOW MACH NUMBER NOZZLE FLOW}

\section{Formulation of the Problem}

An axi-symmetric air-jet of density $\rho_{1}$ and sound speed $c_{1}$ exhausts from a jet pipe of cross-sectional area $A$ through a nozzle of area A into a stationary ambient medium of density and sound speed respectively equal to $\rho_{0}, c_{0}$. (Fig. 1 ). The Mach number of the flow is taken to be sufficiently small that variations in $\rho_{1}, c_{1}$, may be neglected. This will be the case if the steady upstream flow velocity $U$ and the nozzle exit velocity $U_{J}$ satisfy $M^{2}, M_{J}{ }^{2} \ll 1$, where Mach numbers $M, M_{J}$ are defined by

$$
\mathrm{M}=\mathrm{U} / \mathrm{C}_{i}, \mathrm{M}_{\mathrm{J}}=\mathrm{U}_{\mathrm{J}} / \mathrm{C}_{\mathrm{I}}
$$

Dissipation processes will also be neglected, so that for uniform upstream conditions the flow is homentropic, although there may be a variation in the specific entropy $s$ across the mean shear layer of the jet.

A plane harmonic sound wave is incident on the nozzle exit from within the jet pipe. It is required to determine the relation between the flux $W_{T}$, say, of acoustic energy through the nozzle, i.e., through the control surface $\Sigma$ located just upstream of the contraction, and the total acoustic power $W_{F}$ radiated into the ambient medium. Let $p_{I}$ denote the amplitude of the incident wave, such that in the upstream region the incident pressure perturbation is given by the real part of

$$
p=p_{I} e^{i\left\{\frac{k_{1} x_{1}}{1+M}-\omega t\right\}}
$$

In this expression $\omega$ is the radian frequency, $k_{1}=\omega / c_{1}$, $t$ is the time, and the positive direction of the $\mathrm{x}_{1}$-axis of a rectangular coordinate system $\left(x_{1}, x_{2}, x_{3}\right)$ is parallel to the mean flow, the origin being located in the center of the nozzle-exit plane.

The velocity $U$ of the mean flow is a function of position both within the nozzle and in the exterior fluid, and 


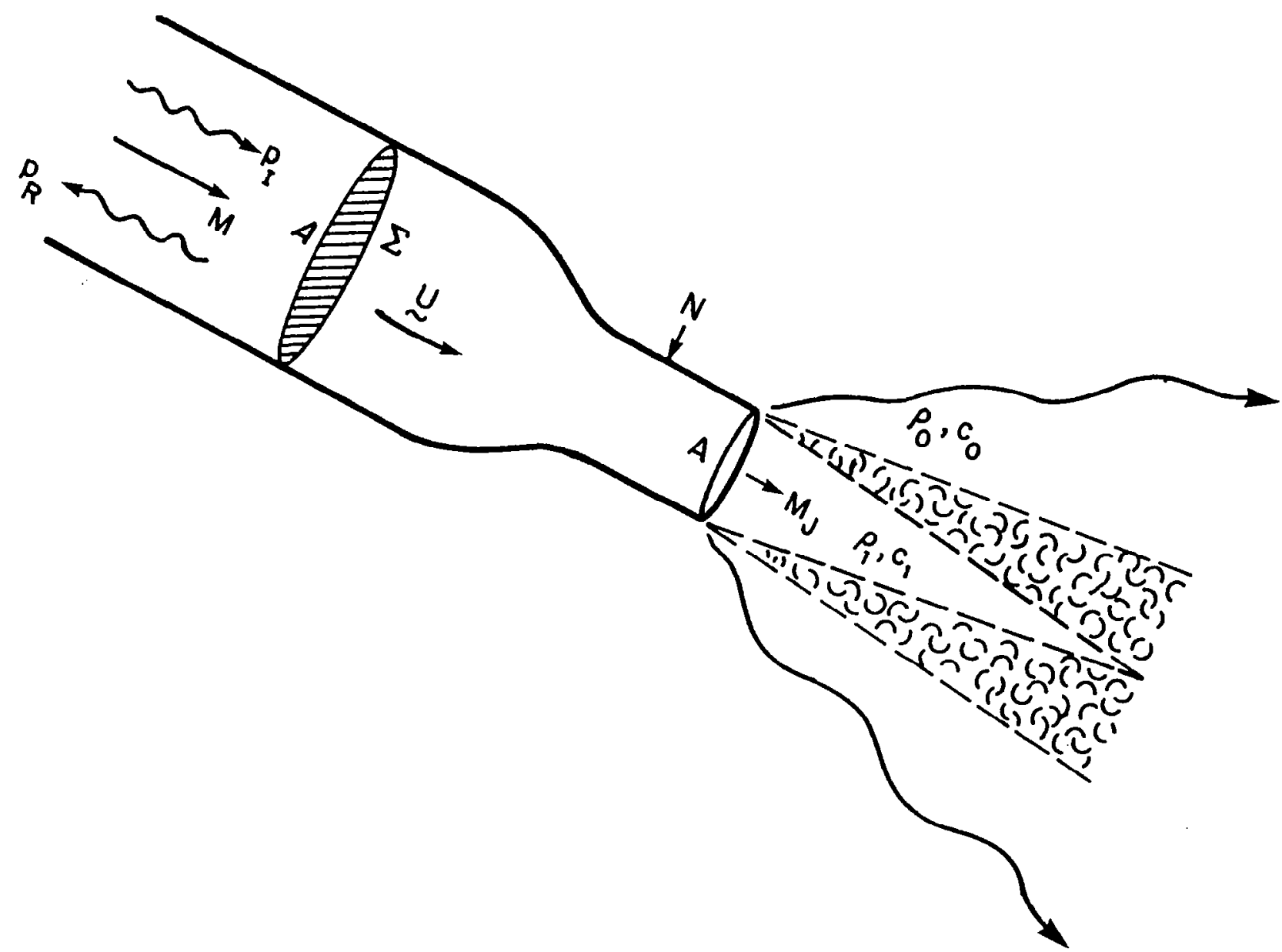

FIG. 1. SCHEMATIC ILLUSTRATION OF THE CONFIGURATION CONSIDERED IN THE ANALYSIS OF THE EMISSION OF LOW FREQUENCY SOUND FROM A JET PIPE IN THE PRESENCE OF A MEAN NOZZLE FLOW. 
in this case the Iighthill (1952) acoustic analogy theory of aerodynamic sound assumes a convenient form when the stagnation enthalpy $B$, rather than the pressure, is taken as the fundamental acoustic variable. The stagnation enthalpy is given in terms of the velocity $\underset{\sim}{v}$ and the specific enthalpy h by

$$
\mathrm{B}=\mathrm{h}+\frac{\mathrm{l}}{2} \mathrm{v}^{2}
$$

In the absence of dissipative processes the inhomogeneous wave equation of the acoustic analogy theory becomes

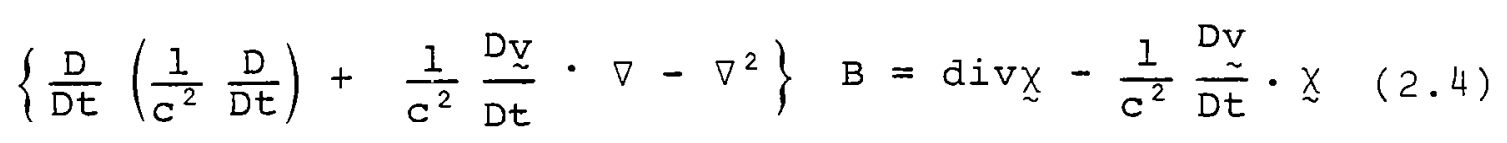

where

$$
\underset{\sim}{\chi}=\underset{\sim}{\omega_{\wedge}} \underset{\sim}{V}-T \nabla S
$$

$D / D t=\partial / \partial t+\underset{v}{v} \cdot \partial / \partial \underset{\sim}{x}, \underset{w}{\omega}=\operatorname{curl} \underset{\sim}{\mathrm{V}}$ is the vorticity, and $\mathrm{T}$ is the temperaturẽ (Howe 1975).

The terms on the right of (2.4) vanish identically except in the shear layer of the jet. The fluid is homentropic in the ambient medium and within and upstream of the potential core of the jet. In those regions the pressure is a function of the density alone, and the specific enthalpy $h$ may be identifed with $\int \mathrm{dp} / \rho$. Similarly, Crocco's form of the momentum equation

$$
\frac{\partial v}{\partial t}+\nabla B=-\underline{x}
$$

(Liepmann \& Roshko 1957, p. 193) reduces to the statement that the flow is irrotational outside of the jet mixing region, with

$$
B=B_{n}-\frac{\partial \phi}{\partial t} \quad(n=0,1) .
$$


Here $\phi$ is the perturbation velocity potential, and $B_{n}$ takes respectively constant values $B_{0}, B_{1}$, say, in the ambient medium and in the potential region of the jet. In free space the acoustic pressure $p$ is given by

$$
\frac{p}{p_{0}}=B^{\prime}
$$

Where $B^{i}=B-B_{0}$.

It follows from these remarks that when the mean flow is disturbed by the incident wave (2.2), a linearized description of the subsequent motion in the potential regions is obtained by setting the variable coefficients of the wave operator on the left of (2.4) equal to their local undisturbed mean values. When terms of $0\left(\mathrm{M}_{J}^{2}\right)$ relative to unity are also discarded the propagation of small disturbances may be taken to be described by the convected wave equation

$$
\left\{\frac{1}{c_{1}^{2}}\left(\frac{\partial}{\partial t}+U_{j} \frac{\partial}{\partial x_{j}}\right)^{2}-\frac{\partial^{2}}{\partial x_{j}^{2}}\right\} B=0
$$

in the potential region of the jet, and by

$$
\left(\frac{I}{c_{0}^{2}} \frac{\partial^{2}}{\partial t^{2}}-\frac{\partial^{2}}{\partial x_{j}^{2}}\right) B=0 \quad,
$$

in the ambient medium.

\section{Energy Flux Within the Jet Pipe}

The flux of acoustic energy through the control surface $\Sigma$ (Fig. 1) into the nozzle may be calculated from the general formula

$$
\mathrm{W}_{\mathrm{T}}=A \cdot\left\{\rho_{1}<\mathrm{UB}^{\prime}>+\mathrm{U}<\rho^{\prime} \mathrm{B}^{\prime}>\right\}
$$


given by Landau and Lifshitz (1959, \$56, 64). The angle brackets denote an average over a wave period $2 \pi / \omega$, $u$ is the perturbation velocity which at $\Sigma$ is parallel to the $\mathrm{x}_{1}$-axis, and $\rho^{\prime}$ is the perturbation density.

Equation (2.3) may be used to express the incident wave (2.2) in the form

$$
\left.\begin{array}{l}
B_{I}^{\prime}=B_{I} e^{i\left(\frac{k_{1} x_{1}}{I+M}-\omega t\right)} \\
B_{I}=(I+M) \frac{P_{I}}{\rho_{1}}
\end{array}\right\}
$$

Let $R$ be a reflection coefficient such that upstream of the nozzle contraction the total perturbation stagnation enthalpy is given by the real part of

$$
B^{\prime}=B_{I}\left\{e^{i \frac{k_{1} x_{1}}{(1+M)}}+R e^{-\frac{i k_{1} x_{1}}{T I-M)}}\right\} e^{-i \omega t}
$$

Equations (2.6), (2.13) and the adiabatic relation between density and pressure may now be used to express the energy flux (2.11) in the form

$$
W_{T}=W_{0}\left(1-|R|^{2}\right)
$$

where $W_{0} \equiv A \rho_{1}\left|B_{I}\right|^{2} / 2 c_{1}$ is the power flux of the incident wave (2.2). This result can be shown to coincide with Blokhintsev's (1946) formula

$$
\mathrm{W}_{\mathrm{T}}=\frac{A}{2 \rho_{1} c_{1}}\left\{(1+M)^{2}\left|p_{I}\right|^{2}-(I-M)^{2}\left|p_{R}\right|^{2}\right\},
$$

where $\mathrm{p}_{R}$ is the amplitude of the reflected pressure perturbation. The reflection coefficient $R$ is determined by the exterior flow properties of the jet contained within the aerodynamic source vector $\underset{\sim}{x}$, and will be discussed in Sec. 3 . 


\section{The Free Space Radiation}

The characteristic acoustic wavelength is assumed to be large compared with the length scale of the nozzle and this, together with the low Mach number restriction, enables the effect of fluid compressibility in the nozzle to be neglected in a first approximation. Broadband fluctuations in the flow produced by nonlinear fluctuations in the aerodynamic source vector $\chi$ of Eq. (2.4) are also ignored. This is justified by the experiments of Bechert, Michel and Pfizenmaier (1977), which reveal negligible amplification of the broadband radiation for moderate amplitude tonal excitation. Thus only that component of $X$ which is directly proportional to the incident wave need be retained.

These approximations permit the replacement of the propagation operator in the acoustic analogy Eq. (2.4) by its standard convected far field form (2.9) [reducing to (2.10) in free space], and also allow the second aerodynamic source term on the right hand side to be discarded, yielding

$$
\left\{\frac{1}{c^{2}}\left(\frac{\partial}{\partial t}+u_{j} \frac{\partial}{\partial x_{j}}\right)^{2}-\frac{\partial^{2}}{\partial x_{j}^{2}}\right\} \quad B=\frac{\partial x_{j}}{\partial x_{j}}
$$

(c.f. Howe 1975).

A formal representation of the solution of this equation in free space may be obtained by making use of the "advanced potential" Green's function $G(\underset{\sim}{x}, \underset{\sim}{y}, t, \tau)$ which satisfies

$$
\left\{\frac{1}{c^{2}}\left(\frac{\partial}{\partial \tau}+U_{j} \frac{\partial}{\partial y_{j}}\right)^{2}-\frac{\partial^{2}}{\partial y_{j}^{2-}}\right\} G=\delta(\underset{\sim}{x}-y) \delta(t-\tau), \quad(2.17)
$$

the condition of vanishing normal derivative on the rigid surface of the nozzle, and corresponds to an implosive sink at $(x, t)$ which vanishes for $\tau>t$. In the absence of mean flow the form of $G(x, y, t, \tau)$ has been given by Ffowcs Williams and Howe (1975) in the compact approximation in which the acoustic wavelength is large compared to the length scale of the nozzle; the modifications required in the present discussion are outlined in the Appendix. 
Equation (2.16) is solved by applying Kirchoff's procedure to (2.16), (2.17) (see, e.g., Stratton 1941, Chapter 8), and for an observer located in free space we find

$$
\begin{aligned}
p / p_{0} \equiv & B(\underset{\sim}{x}, t) \\
& =-\int \underset{\sim}{x} \cdot \frac{\partial G}{\partial \underset{\sim}{y}} d^{3} \underset{\sim}{y} d \tau-\oint_{\Sigma}\left\{\left(G \frac{\partial B}{\partial y_{1}}-B \frac{\partial G}{\partial y_{1}}\right)+\frac{M}{C_{1}}\left(B \frac{D G}{D \tau}-G \frac{D B}{D \tau}\right)\right\} d y_{2} d y_{3} d \tau
\end{aligned}
$$

where the surface integral is taken over the control surface $\Sigma$ of Fig. 1 and $D / D \tau=\partial / \partial \tau+U \partial / \partial y_{1}$. In obtaining this result the momentum equation (2.6) has been used to eliminate contributions from surface integrals over the rigid nozzle. The volume integral is restricted to the shear layer of the jet where $x$ is non-zero, and accounts for the sound produced by vorticity and entropy fluctuations induced by the incident sound wave (2.2).

The contribution $B_{\Sigma}$, say, from the surface integral in (2.18) is evaluated by noting that the radiation condition ensures that only the component of $B$ corresponding to the incident wave (2.2) need be considered. This contribution may be deduced from the representation (A.3) of $G(\underset{\sim}{x}, \underset{\sim}{\mathrm{y}}, \underset{\sim}{t}, \tau)$ given in the Appendix, and is found to be

$$
\mathrm{B}_{\Sigma}(\underset{\sim}{x}, t)=\frac{-i \mathrm{k}_{i} A B_{I}}{2 \pi|\underset{\sim}{x}|}
$$

where $\theta$ is the angle between the positive $x_{1}$-axis and the observer direction, and [ ] denotes evaluation at the retarded time $t-|\underset{\sim}{x}| / c_{0}$.

Similarly, using (A.3) and noting that $x$ must be an axi-symmetric function of position, the volume integral $B_{\underset{\sim}{x}}$
of $(2.18)$ becomes

$$
B_{\underset{\sim}{X}}(x, t)=\frac{i k_{1}\left(1-\frac{c_{1}}{c_{0}} \frac{A}{A} \cos \theta\right)}{4 \pi|\underset{\sim}{x}|} \int\left[x \cdot \frac{\partial^{F_{A}}}{\partial \underset{\sim}{y}}\right] d^{3} y+\frac{i k_{1}\left(\frac{c_{1}}{c_{0}}\right) \cos \theta}{4 \pi|\underset{\sim}{x}|} \int\left[x_{1}\right] d^{3} \underset{\sim}{y}
$$


where the function $\mathrm{F}_{\mathrm{A}}(\mathrm{y})$ is the potential of incompressible, ideal flow from the noz̃zle which has unit velocity in the positive $\mathrm{x}_{1}$-direction upstream of the nozzle contraction. Actually we have neglected a term in (2.20) which is $0(M)$ smaller, M being the Mach number of the upstream flow. This is a valid approximation for large values of the area ratio $A / A$, and in discussing experiments of Bechert, Michel and Pfizenmaier (1977) we shall be concerned principally with a case in which the nozzle exit Mach number $\mathrm{M}_{J}=0.3$ and $\mathrm{M} \simeq 0.04$.

Combining (2.19), (2.20) it follows that in the ambient medium

$\frac{\mathrm{p}}{\rho_{0}}=\frac{-i k_{1} A}{2 \pi|\underset{\sim}{x}|}\left\{\mathrm{B}_{\mathrm{I}} \mathrm{e}^{-i \omega[\mathrm{t}]}-\frac{\left(1-\frac{\mathrm{c}_{1}}{\mathrm{c}_{0}} \frac{\mathrm{A}}{A} \cos \theta\right)}{2 A} \int\left[\underset{\sim}{x} \cdot \frac{\partial^{\mathrm{F}} \mathrm{A}}{\partial \mathrm{y}}\right] \mathrm{d}^{3} \mathrm{y}-{\frac{i c_{1}}{2 c_{0}}}^{\cos \theta} \int\left[x_{1}\right] \mathrm{d}^{3} \underset{\sim}{\mathrm{y}}\right\}$

A complete specification of the radiation depends on the distribution $\underset{x}{x}$ of the aerodynamic sources, which is the subject of the nex section, although it may be anticipated from the definition (2.5) that the contribution from the second term in the brace brackets of $(2.21)$ is $O\left(M_{0}\right) B_{I}$, where $M_{0}=U_{J} / c_{0}$. The expansion of (2.21) in powers of $M_{0} \cos \theta$ and the rejection of terms $\sim 0\left(\mathbb{M}_{0}{ }^{2}\right)$ relative to unity, shows that in the leading approximation the radiation pattern is equivalent to that produced by a monopole-dipole combination, the axis of the dipole being perpendicular to the nozzle exit plant. 


\section{THE FLUX OF ENERGY THROUGH THE NOZZLE}

\section{The Reflection Coefficient}

Consider the fluctuating flow at a point just upstream of the nozzle contraction, which by hypothesis lies well within an acoustic wavelength of the nozzle exit $\left(k_{1} x_{1}<1\right)$. Expand the right hand side of (2.13) in powers of $k_{1} x_{1}$ to obtain

$$
B=B_{I}\left\{(I+R)+i k_{1} x_{1}(I-R)+\ldots\right\} e^{-i \omega t},
$$

in which terms $\sim O\left(k_{1} x_{1} M\right)$ relative to unity have been discarded - M being the upstream Mach number of the flow.

In the incompressible approximation it follows from (2.7) that in the potential region of the jet within the nozzle

$$
B=B_{I}\left\{\alpha+B\left(F_{A}(\underset{\sim}{x})+F_{J}(\underset{\sim}{x})\right)\right\} e^{-i \omega t},
$$

where $\alpha, \beta$ are constant, $F_{A}(x)$ is the potential function introduced in sec. 2 which des̃cribes axi-symmetric flow from the nozzle in the absence of the jet, and $F_{J}(\underset{\sim}{x})$ represents the correction function required to account for the "backreaction" of the exterior jet flow. This back-reaction is given by the causal solution of (2.16) in the incompressible limit :

$$
-\frac{\partial^{2} B_{J}}{\partial x_{j}{ }^{2}}=\frac{\partial x_{j}}{\partial x_{j}}
$$

where

$$
B_{J}=\beta B_{I} F_{J}(\underset{\sim}{x}) e^{-i \omega t}
$$

The behavior of $B_{J}$ upstream of the nozzle contraction is required in order to match the incompressible representation (3.2) with the near field approximation (3.1). This can be 
determined by making use of the corresponding limiting form of the Green's function $G(\underset{\sim}{\mathrm{x}}, \underset{\sim}{\mathrm{y}})$ which satisfies

$$
-\frac{\partial^{2} G}{\partial \dot{y}_{j}^{2}}=\delta(\underset{\sim}{x}-\underset{\sim}{y}),
$$

and the condition of vanishing normal derivative on the rigid surface of the nozzle. When the source point $y$ is within or downstream of the nozzle and the observer location $x$ is in the flow upstream of the contraction, it follows from a simple application of the reciprocal theorm (Rayleigh 1945, Sec. 294) that we may take

$$
G(\underset{\sim}{\mathrm{x}}, \underset{\sim}{\mathrm{y}})=-\frac{1}{A} \mathrm{~F}_{\mathrm{A}}(\underset{\sim}{\mathrm{y}})
$$
Hence forming the convolution product of $G$ and $\partial x_{j} / \partial x_{j}$ we
have in the upstream region

$$
\mathrm{B}_{\mathrm{J}} \rightarrow \frac{1}{A} \int \underset{\sim}{x} \cdot \frac{\partial \mathrm{F}_{\mathrm{A}}}{\partial \underset{\sim}{\underline{y}}} \mathrm{~d}^{3} \underset{\sim}{\mathrm{y}}
$$

an expression which is independent of the upstream location $x$.

This result shows that the reciprocating velocity of the fluid upstream of the nozzle exit is completely described by the potential $\mathrm{F}_{\mathrm{A}}(\mathrm{x})$ of (3.2). In the linearized approximation $x$ must be proportional to this pulsational velocity, i.e., "to $\beta B_{T} e^{-i \omega t}$, and we may accordingly define a complex "hydrodynamic end-correction"

$$
\ell_{H}=\gamma+i \delta,
$$

where $\gamma, \delta$ are determined by incompressible properties of the exterior shear flow. Upstream of the contraction we then have

$$
B_{J}=-\beta B_{I}(\gamma+i \delta) e^{-i \omega t} \equiv \frac{1}{A} \int \underset{\sim}{\chi} \cdot \frac{\partial F_{A}}{\partial \underset{\sim}{y}} d^{3} \underset{\sim}{y},
$$

where real parts are to be taken. 
The constant $\alpha$ in (3.2) accounts for the possibility of a periodic variation in the fluid pressure which is uniform throughout the whole of the incompressible nozzle flow region, and is associated with the monopole component of the radiation into the ambient medium given by (2.21). Let this monopole be represented by

$$
\underset{\rho_{0}}{p}=\frac{\Phi_{\mathrm{m}}}{|\underset{\sim}{\mathbf{x}}|} e^{-i \omega\left(t-\frac{|\underline{\mathbf{x}}|}{\mathrm{c}_{0}}\right)}
$$

for large $|x|$. At distances $|x|$ well within a wavelength of the nozzle Eut large compared with the nozzle exit radius a, this expression may be expanded in powers of the retarded time:

$$
\frac{p}{\rho_{0}}=\frac{\Phi_{m}}{|\underset{\sim}{x}|} e^{-i \omega t}+i k_{0} \Phi_{m} e^{-i \omega t}+\ldots \text {, }
$$

where $k_{0}=\omega / c_{0}$. The first term on the right describes a pulsation in fluid volume through a spherical control surface centered on the nozzle exit, and must equal that produced by the potential $\mathrm{F}_{\mathrm{A}}(\mathrm{x})$ of $(3.2)$. Noting that $\partial \mathrm{F}_{\mathrm{A}} / \partial \mathrm{x}_{1} \rightarrow 1$ as $x_{1} \rightarrow-\infty$ within the jet pipe, we therefore have

$$
\beta_{I^{A}}=-4 \pi \Phi_{\mathrm{m}}
$$

The second term on the right of (3.II) generates a uniform fluctuation in pressure which matches that within the nozzle provided that

$$
\alpha \rho_{1} B_{I}=i \rho_{0} k_{0} \Phi_{m}
$$

Eliminating $\Phi_{m}$ from these relations we find:

$$
\alpha=-\frac{i \beta}{4 \pi}\left(\frac{\rho_{0}}{\rho_{1}}\right) \mathrm{k}_{0} A
$$


We are now ready to equate corresponding terms of the representations ( 3.1$),(3.2)$ of the acoustic field, and thereby determine the reflection coefficient $R$ in terms of the nozzle exit conditions. To do this first observe that upstream of the contraction $F_{A}(\underset{\sim}{x}) \simeq x_{1}-\lambda$ (see Appendix), where the added length $\lambda$ is real and is proportional to the sum of the Helmholtz organ pipe end-correction (Rayleigh 1945, Chapter 16) and a component arising from the increased resistance to flow produced by the contraction.

Thus letting $x_{1} \rightarrow-\infty$ in (3.2) and identifying terms in the resulting expression with corresponding members of (3.1), we obtain:

$$
\left.\begin{array}{c}
\beta=i k_{1}(1-R) \\
\beta\left\{\Lambda+i\left(\delta+\frac{k_{0} A \rho_{0}}{4 \pi \rho_{1}}\right)\right\}=-(I+R)
\end{array}\right\}
$$

use having been made of (3.9), (3.14), and where we have set

$$
\Lambda=\lambda+\gamma
$$

Hence solving for $R$ we find

$$
R=-\left(\frac{1-k_{1}\left(\delta+\frac{k_{0} A \rho_{0}}{4 \pi \rho_{1}}-i \Lambda\right)}{1+k_{1}\left(\delta+\frac{k_{0} A \rho_{0}}{4 \pi \rho_{1}}-i \Lambda\right)}\right)
$$

and using this expression in Eq. (2.14) it follows that the flux of energy from the nozzle can be expressed in the form:

$$
\frac{W_{0}\left(\frac{A}{A}\right)\left\{\frac{\Delta k_{1} \delta A}{A}+\left(\frac{\rho_{0} C_{0}}{\rho_{1} C_{1}}\right)\left(k_{0} a\right)^{2}\right\}}{\left.\left.\frac{4 k_{1} \delta A}{A}+\left(\frac{\rho_{0} C_{0}}{\rho_{1} C_{1}}\right)\left(k_{0} a\right)^{2}\right]\right)^{2}+\left(k_{1} \Lambda\right)^{2}}
$$


This result shows that the flux of energy into the nozzle from the incident sound wave (2.2) is determined by two factors, corresponding to each of the terms in the brace brackets of the numerator. The first depends on the imaginary part of the hydrodynamic end-correction $\ell_{H}$, and is entirely a feature of incompressible properties of the exterior jet flow. The second, proportional to $\left(k_{0} a\right)^{2}$, arises from the monopole component of the sound radiated into the ambient medium. There is no explicit contribution from the dipole component of $(2.21)$ because it is automatically contained within the hydrodynamic term of (3.18).

\section{The Mechanism of Hydrodynamic Attenuation}

An appreciation of the mechanism by which the exterior jet flow extracts energy from the acoustic field may be obtained from a consideration of the contribution of the back-reaction $B_{J}$ of the shear flow to the general energy flux formula (2.11), and we shall do this before proceeding to applications of the above results to specific modelings of the shear flow.

In the present approximation only the first ('incompressible') term in the parentheses of (2.II) need be retained. When the pulsatile nozzle flow is normalized with respect to the coefficient $\beta$ of (3.2) it follows from our earlier discussion that the axial perturbation velocity $u$ depends only on the geometrical configuration of the nozzle [i.e., on $\left.F_{A}(x)\right]$ and not on the properties of the exterior flow. Hence (2.II) implies that the exterior shear flow induces an additional energy flux $W_{J}$, say, through the nozzle, where

$$
\mathrm{W}_{\mathrm{J}}=A \rho_{1}<\mathrm{uB}_{\mathrm{J}}>
$$

Now $\partial \mathrm{F}_{\mathrm{A}} / \partial \mathrm{x}_{1} \simeq$ I upstream of the nozzle contraction, so that the local fluctuating velocity $\mathrm{V}_{\mathrm{A}}$ that would be produced by the acoustic field in the exterior fluid in the absence of the jet is just

$$
{\underset{\sim}{\mathrm{V}}}_{\mathrm{A}}=\mathrm{u} \nabla \mathrm{F}_{\mathrm{A}}
$$


Equations (3.9), (3.19) therefore show that

$$
\begin{aligned}
& w_{J}=\int \underset{\sim A}{v} \cdot \rho_{1} \underset{\sim}{x} d^{3} \underset{\sim}{y}
\end{aligned}
$$

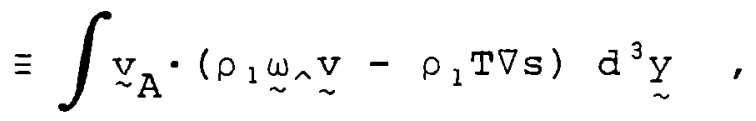

which states that the power flux through the nozzle induced by the essentially incompressible properties of the exterior flow is proportional to the rate of working of the aerodynamic dipole $\underset{\sim}{x}$ in the acoustic component $\underline{\sim}_{A}$ of the fluctuating velocity field. Note that the "lift" experienced by a vortex element is equal to - $\omega_{n} \underset{\sim}{ }$ per unit mass, and the specific "inertia" force $T \nabla s$ is ẽqual to $-p \nabla(I / \rho)$ in the absence of dissipation, and represents the reaction of a fluid particle when accelerated in an environment of different density.

A particularly illuminating form of (3.21) emerges in the case of uniform mean density. The "inertia" force vanishes identically, and in the incompressible limit

$$
\underset{\sim}{(\omega, \underset{\sim}{v})_{i}}=\frac{\partial}{\partial x_{j}}\left(v_{i} v_{j}\right)-\frac{\partial}{\partial x_{i}}\left(\frac{1}{2} v^{2}\right)
$$

Integrate ( 3.21 ) by parts, and observe that there is no contribution from the resulting integral over the surface of the nozzle, to obtain

$$
w_{J}=-\int \varepsilon_{i j} \rho_{0} v_{i} v_{j} d^{3} \underset{\sim}{y} \quad,
$$

where

$$
\varepsilon_{i j}=\frac{1}{2}\left(\frac{\partial v_{A i}}{\partial x_{j}}+\frac{\partial v_{A j}}{\partial x_{i}}\right)
$$

is the tensor rate of strain produced by the acoustic component of the fluctuating nozzle flow. The velocity $v_{i}$ in (3.22), (3.23) may be regarded as the total velocity minus the acoustic component $\tilde{\sim}_{A}$, since curl $\tilde{v}_{A} \equiv 0$ and the contribution $-\omega_{A} V_{A}$ to the Iift involves the perfformance of no work. Thus the rate at which energy exhausts from the nozzle 
due to the excitation of incompressible disturbances in the exterior jet flow is just equal to the rate at which work is performed on the exterior fluld by the Reynolds stress

system- $\rho_{0} v_{j} v_{j}$ of the vortical flow in the rate of strain field of the acoustic disturbance. The energy provided in this way is used to generate vorticity at the lip of the nozzle and may thereby maintain a steady system of spatial instability waves on the jet. The latter may be identified with the incipient form of the "coherent structures" observed by Moore (1977) and others.

A preliminary quantitative estimate of the magnitude of the hydrodynamic attenuation is readily obtained in the limit of small Strouhal number wa/U $U$. Let $u_{A}$ denote the amplitude of the mean axial component of the fluctuating jet velocity in the nozzle exit plane. Reference to (3.2) and use of the

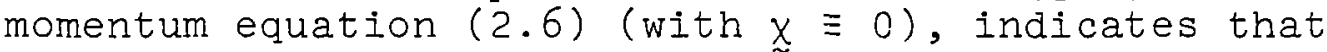

$$
u_{\mathrm{A}}=-i \frac{A B B_{I}}{\bar{A} \omega}
$$

the factor $A / A$ arising from the continuity of flow in the nozzle. The nozzle fluctuations produce a periodic train of axi-symmetric vortex rings whose circulation per unit length in the $x_{1}$-direction is equal to $U_{J}+u_{A} e^{-i \omega t}$ at the nozzle exit, and which convect downstream at velocity $\left(U_{\mathrm{J}}+u_{A} e^{-i \omega t}\right) / 2$ (cf. Saffman 1975). If the mean shear layer close to the nozzle exit is not too thick, it follows in a linearized approximation that

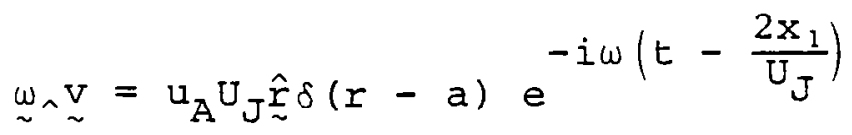

where the radial coordinate $r=\sqrt{x_{2}{ }^{2}+x_{3}{ }^{2}}$ and $\hat{r}$ is the corresponding unit vector.

In the case of a cold jet, for which $\rho_{1} \equiv \rho_{0},(3.26)$ is the only contribution to the aerodynamic source vector $x$ of (2.5) and its substitution into equation (3.9) shows thãt:

$$
\gamma+i \delta=\frac{i U_{J}}{A \omega} \oint_{\nabla F_{A}} \cdot d S_{\sim} e^{\frac{2 i \omega Y_{1}}{U_{J}}}
$$


where the surface integral is taken over the nominal boundary $r=a$ of the jet. At sufficiently small strouhal numbers the variation of the exponential in the integrand may be neglected over that portion of the boundary where $\nabla F_{A}$ is significant, and it then follows from the definition of $\mathrm{F}_{\mathrm{A}}$ that

$$
\gamma=0 ; \delta=\frac{A U_{\mathrm{J}}}{\mathrm{A} \omega},
$$

a result which illustrates the dependence of the end-correction $\ell_{\mathrm{H}}$ on the hydrodynamic length scale $\mathrm{U}_{\mathrm{J}} / \omega$ of the fluctuating nozzle flow.

Inserting these values of $\gamma, \delta$ in equation (3.17) we find that the reflection coefficient $R$ has the explicit form

$$
R=-\left(\frac{1-\frac{A}{A}\left(M_{J}+\frac{\left(k_{0} a\right)^{2}}{4}\right)+i k_{0} \lambda}{1+\frac{A}{A}\left(M_{J}+\frac{\left(k_{0} a\right)^{2}}{4}\right)-i k_{0} \lambda}\right),
$$

in which $k_{0}=k_{1}$ for a cold jet. Similarly the energy flux $W_{T}$ becomes:

$$
W_{T}=\frac{W_{0}\left(\frac{A}{A}\right)\left\{4 M_{J}+\left(k_{0} a\right)^{2}\right\}}{\left(1+\frac{A}{4 A}\left[4 M_{J}+\left(k_{0} a\right)^{2}\right]\right)^{2}+\left(k_{0} \lambda\right)^{2}}
$$

These approximate expressions, which are nonetheless characteristic of the general case, show that in the presence of the mean nozzle flow, the limit of long wavelength $\left(k_{0} a, k_{0} \lambda \rightarrow 0\right)$ does not reproduce the classical results $R=-I, W_{T}=0$ for reflection at an open end. Indeed for sufficiently large area ratio $A / A$, it is clear that $R \cong+I$ and $\mathrm{W}_{\mathrm{T}} \simeq 4 \mathrm{~W}_{\mathrm{O}} \mathrm{A} / A \mathrm{M}_{\mathrm{J}}$. 


\section{Exterior Flow Models}

A precise determination of the complex hydrodynamic endcorrection $\ell_{H}=\gamma+$ is, and thence of the energy flux $W_{T}$, involves the analysis of a specific modeling of the exterior shear flow. This is facilitated by the assumption that the nozzle possesses a circular cylindrical neck which extends a distance of at least one nozzle exit radius downstream of the contraction as in Fig. 1. It may then be asserted that local details of the exterior incompressible flow do not depend critically on upstream variations in nozzle geometry, an hypothesis which is justified by the observation that the back-reaction $B_{J}$ produces no additional velocity fluctuations in the upstream region. We shall therefore examine shear flows calculated on the basis of pulsatile incompressible flow from a semi-infinite, circular cylindrical duct.

Two cases I, II will be discussed. In case I the boundary of the jet is represented by a linearly disturbed vortex sheet. A thorough discussion of this problem for compressible flow has been given by Munt (1977), and a statement of the relevant results obtaining in the incompressible limit is therefore sufficient for our purposes. Additional details are outlined in the Appendix.

In Munt's theory the Kutta condition of finiteness is imposed at the nozzle lip. This is presumably appropriate at the relatively low Strouhal numbers of interest in the present discussion (c.f., Bechert and Pfizenmaier 1975 b); at higher frequencies and correspondingly smaller length scales, sound emerges from the duct without "feeling" the lip, and propagates along energy conserving ray paths through the mean shear layer. The shear layer is unstable, however, the instabilities being associated with eigenmodes of oscillation of the jet, and Munt obtains a strictly causal solution in which these modes are triggered and sustained by the flucuating nozzle flow.

Taking the incompressible limit in Munt's theory (see Appendix) the hydrodynamic end-correction $l_{\mathrm{H}}=\gamma+i \delta$ can be expressed in the following form in

Case I:

$$
\begin{aligned}
& \gamma=\frac{A U_{J}}{A \omega}(\zeta-\mu), \\
& \delta=\frac{A}{A} \frac{U_{J}}{\omega} v
\end{aligned}
$$


The dimensionless quantities $\zeta, \mu, \nu$ are real functions of the Strouhal number wa/ $\mathrm{U}_{J}$ and the density ratio $\rho_{0} / \rho_{1}$, and are defined by means of the dispersion function

$$
\mathrm{z}\left(\frac{\mathrm{U}_{\mathrm{J}}^{\mathrm{k}}}{\omega}, \mathrm{ka}, \frac{\rho_{\Omega}}{\rho_{1}}\right)
$$

given in equation (A.7) of the Appendix, whose zeros determine those axi-symmetric incompressible eigenmodes of a circular cylindrical jet which are proportional to $\exp \left\{i\left(k x_{1}-\omega t\right)\right\}$. When $\omega$ is real and positive, say, the zero $k=k_{I}$ of $z$ corresponding to the spatially growing instability mode of the jet lies in the fourth quadrant of the $k$-plane, and defines $\mu, v$ by

$$
\nu+i \mu=\frac{\omega}{U_{J} k_{I}}
$$

The imaginary part $\mu$ tends to zero as wa/ $\mathrm{U}_{J} \rightarrow 0$. The real part $v$ determines the attenuation of the incident sound due to the excitation of the instability wave on the jet, and

$$
\left.\begin{array}{l}
\nu \rightarrow 1 \text { as } \omega a / U_{J} \rightarrow 0 \\
\rightarrow \frac{\rho_{1}}{\rho_{1}+\rho_{0}} \text { as } \omega a / U_{J} \rightarrow \infty
\end{array}\right\}
$$

and varies monotonically between these limits for intermediate values of the Strouhal number.

The remaining function $\zeta$ is given in terms of $z$ by

$$
\zeta=\frac{1}{2 \pi} \int_{-\infty}^{\infty} \frac{\ln \left|\mathrm{z}\left(\xi, \xi\left(\frac{\omega \mathrm{a}}{\mathrm{U}_{J}}\right), \frac{\rho_{0}}{\rho_{1}}\right)\right| d \xi}{\xi^{2}}
$$

and tends to zero with the Strouhal number wa/ $\mathrm{U}_{\mathrm{J}}$.

Pinker and Bryce (1976) and Savkar (1975) suggest that in practice instability waves are not excited at low subsonic Mach numbers. This would be expected to be the case if the 
width of the shear layer were sufficiently large with respect to the hydrodynamic length scale of the fluctuating flow. We shall therefore examine the opposite extreme to that discussed by Munt (1977), and assume in Case II that the effect of the finite width of the shear layer cannot be neglected.

An approximate analytical treatment of this case may be given by noting that in the experiments of Pinker and Bryce (1976), the width of the shear layer at the nozzle exit is substantailly greater than the anticipated viscous controlled width $\sim \sqrt{v_{A} / \omega}$ of vorticity shed from the nozzle lip ( $\nu_{A}$ being the kinematic viscosity $\sim 1.5 \times 10^{5} \mathrm{~m}^{2} / \mathrm{s}$ for air at $300^{\circ} \mathrm{K}$ ) even at the lowest frequency of interest. Thus if attention be confined to a cold jet, in the linearized approximation the aerodynamic source dipole becomes

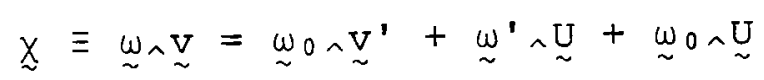

where $\mathrm{V}^{\prime}, \underset{\sim}{\omega}$ 'respectively denote the perturbation velocity and vorticity, and $U, w_{0}$ are the corresponding mean flow quantities. The back-reaction BJ therefore satisfies the following approximate form of (3.3):

$$
\left.-\nabla^{2} B_{J}=\operatorname{div} \underset{\sim}{(\underset{\omega}{\prime}} \sim_{\sim}^{U}\right)
$$

provided that the length scale of the shed vorticity is small compared with the width of the shear layer. In this case the shed vorticlty is located within the shear layer in a region of effectively uniform mean velocity and convects downstream at this velocity which is well approximated by one half of the nominal center-line jet velocity $\mathrm{U}_{\mathrm{J}}$ (Davies, Fisher and Barrat 1963). The vorticity $\omega^{\prime}$ is assumed to convect along the mean boundary $r=a$ of thẽ jet and its strength is determined via the Kutta condition. The procedure is outlined in the Appendix, and leads to the following determination of the hydrodynamic end-correction in Case II:

$$
\gamma=0 ; \delta=\frac{A U_{J}}{2 A \omega}
$$

Return now to the general energy flux formula (3.18). Using the above results for the hydrodynamic end-correction we obtain the following predictions for the total perturbation energy flux from the nozzle: 
Case I: Vortex Sheet Model:

$$
W_{T}=\frac{W_{0}\left(\frac{A}{A}\right)\left\{4 M_{J} \nu+\frac{\rho_{0} C_{0}}{\rho_{1} C_{1}}\left(k_{0} a\right)^{2}\right\}}{\left(1+\frac{A}{4 A}\left[4 M_{J} \nu+\frac{\rho_{0} C_{0}}{\rho_{1} C_{1}}\left(k_{0} a\right)^{2}\right]\right)^{2}+\left(k_{1} \lambda+\frac{A}{A} M_{J}(\zeta-\mu)\right)^{2}}
$$

Case II: Finite Width Shear Layer (Cold Jet):

$$
\mathrm{W}_{\mathrm{T}}=\frac{\mathrm{W}_{0}\left(\frac{A}{\mathrm{~A}}\right)\left\{2 \mathrm{M}_{\mathrm{J}}+\left(\mathrm{k}_{0} a\right)^{2}\right\}}{\left(1+\frac{A}{4 A}\left[2 \mathrm{M}_{\mathrm{J}}+\left(\mathrm{k}_{0} a\right)^{2}\right]\right)^{2}+\left(\mathrm{k}_{1} \lambda\right)^{2}}
$$

Observe that the formula (3.38) for case I reduces to the approximation (3.30) in the limit of small Strouhal number (when $\nu \rightarrow I, \zeta, \nu \rightarrow 0$ ) for a cold jet. Interestingly enough, we also see that, again for a cold jet, the limiting value $v \sim 0.5$ as $w a / U_{J} \rightarrow \infty$ implies that the vortex sheet model (3.38) reduces to the finite width shear layer model (3.39). 


\section{THE RADIATED SOUND POWER: COMPARISON WITH EXPERIMENT}

The substitution of (3.9) and the explicit linearized form of $x_{1}$ into equation $(2.21)$ enables the sound field to be expressed in the following form:

$$
\frac{p}{\rho_{0}}=\frac{-i k_{1} A}{2 \pi|\underset{\sim}{x}|}\left\{\left(1+\frac{B}{2}(\gamma+i \delta)\left(I-\frac{c_{1} A}{c_{0} A} \cos \theta\right) B_{I} e^{-i \omega[t]}+\frac{c_{1} \cos \theta}{2 c_{0}} \int\left(\omega_{0} v_{r} d^{3} \underset{\sim}{y}\right\}\right.\right.
$$

where $v_{r}$ is the radial component of the perturbation velocity. In the case of the vortex sheet model the mean vorticity $\omega_{0}$ forms a singular distribution on $r=a$, and the integration in (4.1) reduces to the determination of the volume flux through the nominal boundary of the jet. Since compressible effects are unimportant in the nozzle region this flux is equal to the volume flux from the nozzle, and in the approximation of (3.1) the Integrated term of (4.I) becomes $\frac{1}{2} B_{T}(I-R) M_{0} \cos \theta e^{-i \omega[t]}$. This result would also be expected to be valid to a good approximation for a shear layer of finite width, because the principal contribution to the integral is from the region close to the nozzle where the shear layer is relatively thin. Hence using this, and equations $(3.15 \mathrm{a}),(3.17)$ to calculate $\beta$, it follows that in the approximation of long wavelength the intensity of the free space radiation becomes:

Case I:

$\frac{\left\langle p^{2}\right\rangle}{\rho_{0} c_{0}}=\frac{w_{0}\left(\frac{A}{A}\right)\left(\frac{\rho_{0} C_{0}}{\rho_{1} C_{l}}\right)\left(k_{0} a\right)^{2}}{4 \pi|\underline{x}|^{2}}\left\{\frac{\left(I+(I+v) M_{0} \cos \theta\right)^{2}+M_{0}^{2}(\zeta-\mu)^{2} \cos ^{2} \theta}{\left(1+\frac{A}{A} M_{J} U\right)^{2}+\left(\frac{A}{R}\right)^{2} M_{J}^{2}(\zeta-\mu)^{2}}\right\}$

Case II: (cold jet)

$$
\frac{\left\langle p^{2}\right\rangle}{\rho_{0} c_{0}}=\frac{W_{0}\left(\frac{d}{A}\right)\left(k_{0} a\right)^{2}}{4 \pi|x|^{2}}\left\{\frac{1+\frac{3 M_{0}}{2} \cos \theta}{1+\frac{A M_{J}}{A 2}}\right\}^{2}
$$

When $A=A$ (no nozzle contraction) the vortex sheet Case I agrees with the corresponding limiting form of Munt's (1977) exact analysis. 
The directivities predicted by these formulae are illustrated in Fig. 2 and compared with the field shape data of Pinker and Bryce (1976). That data is for a cold jet with $\mathrm{M}_{0} \equiv \mathrm{M}_{J}=0.3$, and the curve in Case $I$ has been calculated for a Strouhal number wa/U $\mathrm{U}_{J}=0.8$ which corresponds to the experiment at the Helmholtz number $\mathrm{k}_{0} \mathrm{a}=0.24$. Actually the comparison with experiment is probably relevant only for $\theta$ in the range $60^{\circ}-120^{\circ}$, say, where $\cos ^{2} \theta$ is not particularly significant, since our analysis has systematically neglected terms $\sim 0\left(\mathrm{M}_{0}{ }^{2}\right)$ relative to unity. The data has been matched with the theory at $\theta=90^{\circ}$, and for the above range in $\theta$ both models exhibit a tolerable representation of the experimental results, although the overall agreement is better for the vortex sheet model of Case $I$. Note however, that there exists an absolute difference in the levels predicted by the two models at $90^{\circ}$, caused principally by the presence of the area ratio $A / A$ in the denominator of each of (4.2), (4.3). In the Pinker and Bryce experiment $A / A \simeq 3.7$, and this implies that Case II exceeds Case I by about $2.5 \mathrm{~dB}$ at $\theta=90^{\circ}$. This is the only way in which the area ratio $A / A$ influences the radiated sound field, and presumably accounts for the good agreement with experiment of Munt's (1977) field shape predictions based on a circular cylindrical nozzle.

Integration of (4.2), (4.3) over the surface of a large sphere of radius $|\mathrm{x}|$ centered on the nozzle exit yields the total radiated sound power $W_{F}$. The contribution from the dipole component of the field is now $0\left(\mathrm{M}^{2}{ }^{2}\right)$ relative to that of the monopole, and this must be rejected in order to be consistent with our previous approximations. In the limit of long wavelength we then find by comparison with the equations (3.8), (3.9) giving the energy flux $W_{T}$ through the nozzle:

Case I:

$$
\frac{W_{F}}{W_{T}}=\frac{\left(\frac{\rho_{0} c_{0}}{\rho_{1} c_{l}}\right)\left(k_{0} a\right)^{2}}{4 M_{J} \nu+\left(\frac{\rho_{0} c_{l}}{\rho_{1} c_{1}}\right)\left(k_{0} a\right)^{2}}
$$




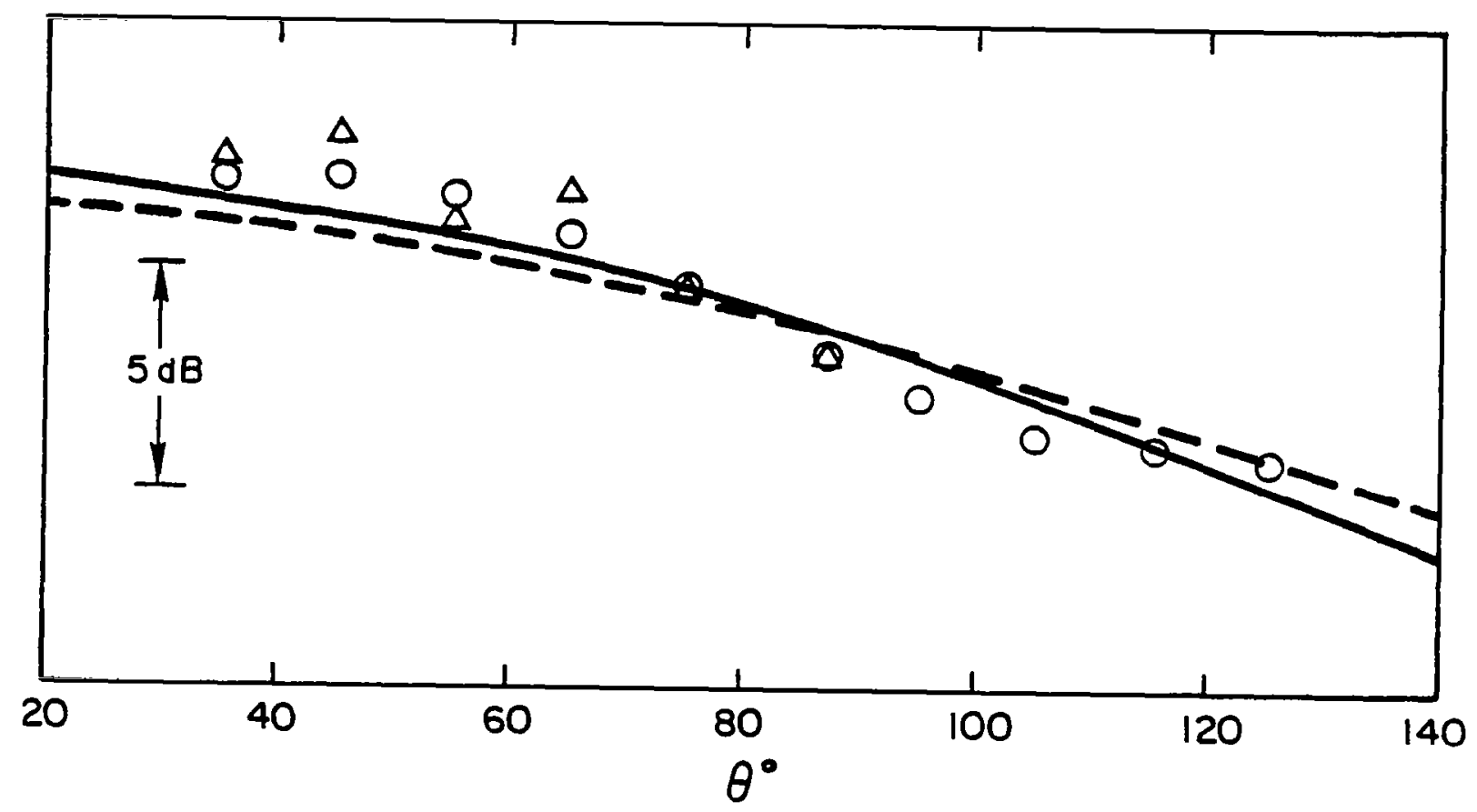

FIG. 2. PREDICTED FIELD SHAPE CHARACTERISTICS FOR ka $=0.24$ AND $M_{J}=0.3:$ CASE I $\longrightarrow$, VORTEX SHEET MODEL;

CASE II - -..., FINITE WIDTH SHEAR LAYER MODEL. THE ANGLE $\theta$ IS MEASURED FROM THE DOWNSTREAM DIRECTION OF THE JET AXIS, AND THE EXPERIMENTAL POINTS ARE TAKEN FROM PINKER AND BRYCE (1976): $\Delta-\mathrm{ka}=0.24 ; 0-\mathrm{ka}=0.6 \mathrm{AT}$ $M_{J}=0.3$. 
Case II (cold jet):

$$
\frac{W_{F}}{W_{T}}=\frac{\left(k_{0} a\right)^{2}}{2 M_{J}+\left(k_{0} a\right)^{2}}
$$

These results are independent of the area ratio $A / A$.

Bechert, Michel and Pfizenmaier (1977) have measured the attenuation $10 \log _{10}\left(W_{F} / W_{T}\right) d B$ in the case of a cold jet over a range of subsonic nozzle exit Mach number $\mathrm{M}_{J}$, the area ratio $A / A$ being equal to 7.6 . Their results are shown in Fig. 3. Figure 4 illustrates the comparison of the predictions (4.4), (4.5) of Cases I, II with the particular low Mach number case $\mathrm{M}_{J} \equiv \mathrm{M}_{0}=0.3$. Both of the theoretical curves predict identical overestimates of the attenuation at the higher values of ka, for which $\nu \sim 0.5$, but of course the compact approximation used in deriving our results would be expected to fail in this region. At lower values of ka the finite shear layer model produces a marginally better agreement with experiment. In any event the agreement with experiment is sufficiently good to give confidence in the validity of the hydrodynamic attenuation mechanism, and indicates that at low frequencies the details of both the attenuation levels and the radiation directivities are relatively insensitive to the precise modeling of the exterior nozzle flow. 


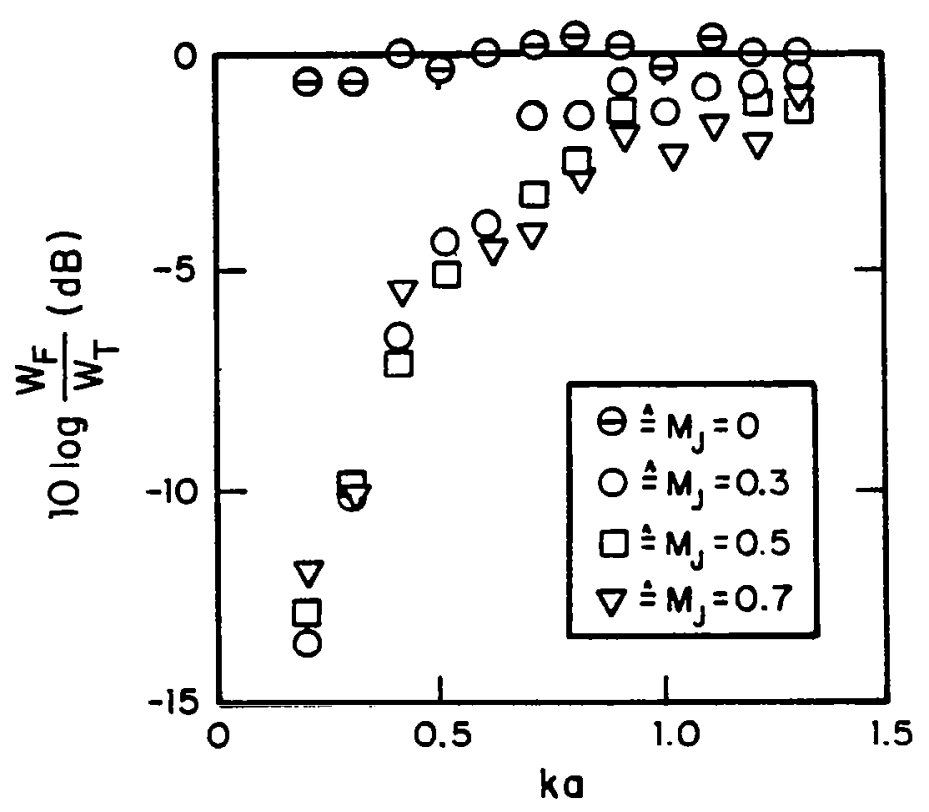

FIG. 3. MEASURED RATIO OF THE FAR FIELD SOUND POWER $W_{F}$ TO THE NOZZLE POWER FLUX W AS A FUNCTION OF THE NOZZLE EXIT HELMHOLTZ NUMBER Ka FOR VARIOUS VALUES OF JET MACH NUMBER MJ (BECHERT, ET AL. 1977). 


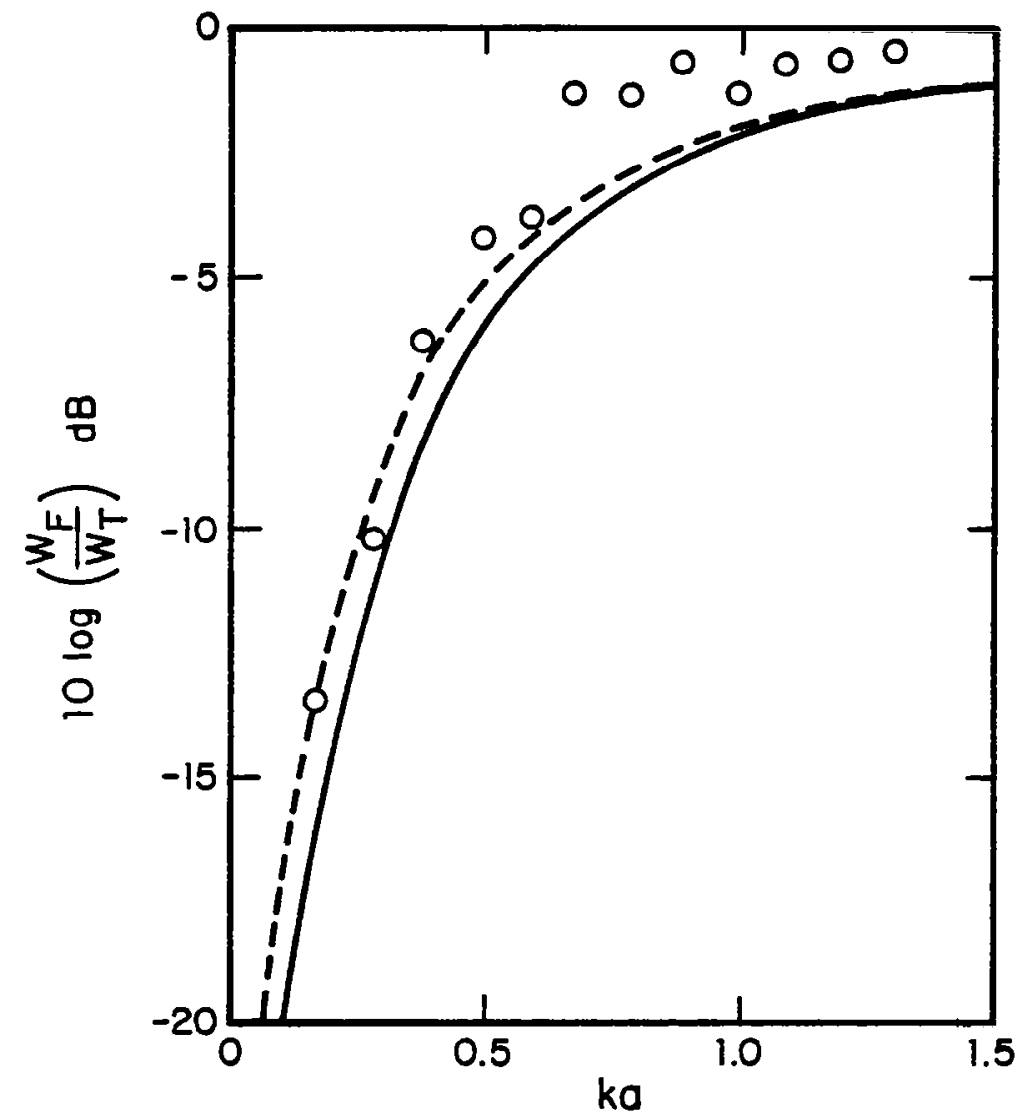

FIG. 4. COMPARISON OF PREDICTED AND MEASURED RATIO $W_{F} / W_{T}$ AS A FUNCTION OF ka FOR $M_{J}=0.3$. EXPERIMENT: 000 (BECHERT ET AL. 1977); THEORY:- CASE I, VORTEX SHEET MODEL, -..-- CASE II, FINITE WIDTH SHEAR LAYER MODEL . 


\section{CONCLUSION}

The emission of low frequency sound from a jet pipe in the presence of a subsonic nozzle flow involves a transfer of energy from the acoustic wave to essentially incompresible vortex waves on the jet. This produces a net attenuation in the transmitted sound which is not compensated by the coherent and/or broadband aerodynamic sound subsequently radiated by the vortex motions. At low Mach number and Helmholtz number ka the predicted attenuation and the field shape of the radiated sound do not depend critically on the details of the theoretical modeling of the exterior flow, although a vortex sheet model tends to predict a slightly lower overall level of radiation than one which incorporates the effects of the finite width of the shear layer. This supports the view that, as far as the interaction with the nozzle is concerned, the question of whether or not the exterior flow is stable is quite irrelevant, because the principal interaction occurs within one hydrodynamic length scale from the lip of the nozzle. The Kutta condition plays a much more significant role in as much as shed vorticity provides both the vehicle by which hydrodynamic energy is conveyed downstream, and also, through its interaction with the nozzle, is responsible for the production of the aerodynamic component of the radiated sound.

The presence of hydrodynamic attenuation at low frequencies implies that the flow of energy through the nozzle attains a minimum over a fairly well-defined range of frequencies, since power transmission is high also at high frequencies due to direct radiation. This could be a significant aspect governing the excitation of duct resonances. The existence of such a minimum was noted by Mechel, Merlins and Schilz (1967), who mistakenly attributed the increased energy flux at low frequencles to a greater radiation efficiency. In this connection a rough estimate of the practical importance of hydrodynamic attenuation at low frequencies may be obtained from a consideration of Howe's (1975) treatment of the aerodynamic generation of standing waves in a pipe of length $\ell$ and cross-section $A$ which is open at both ends. An air jet of cross-section $A_{E}$ and Mach number $\mathrm{M}_{F}$ impinges on a sharp edge of the opening at one end of the pipe, and standing waves of frequency $\omega=n \pi c / \ell$ are excited, where $n$ is the largest integer satisfying

$$
\mathrm{M}_{\mathrm{E}}>\frac{44 A}{A} \sum_{\mathrm{m}} \Delta_{\mathrm{m}}(\mathrm{n}),
$$


$\Sigma$ denoting a sum over frequency dependent terms arising from the various dissipative mechanisms involved. These include radiation from the two open ends, irreversible processes in the boundary layer at the walls of the tube, and, in the present instance, hydrodynamic attenuation produced by the mean flow from the remote end of the pipe. The latter may be shown to correspond to $\Delta=\omega \delta / c$, where $\delta$ is the imaginary part of the hydrodynamic end correction. Using the finite shear layer model of Sec. 3, and assuming that half of the air from the incident jet passes down the pipe [so that $\left.M_{J} \simeq M_{E}\left(A_{E} / 2 A\right)\right]$, it follows from (5.1) that

$$
M_{E}>\frac{\frac{44 A}{A} \sum_{m}^{\prime} \Delta_{m}(n)}{1-11\left(\frac{E}{A}\right)^{2}},
$$

where $\Sigma$ ' denotes the summation excluding the hydrodynamic term. The demoninator in this result would be replaced by unity in the absence of hydrodynamic attenuation. Thus, using the value $A_{E} / A \simeq 0.25$ (appropriate for a descant recorder with all finger holes closed), we see that hydrodynamic attenuation increases by more than a factor of 3 the Mach number $\mathrm{M}_{\mathrm{E}}$ required to excite the nth mode.

In the case of the jet pipe of an aeroengine, Bechert, Michel and Pfizenmaier (1977) point out that the large hydrodynamic attenuation observed in their experiment at low frequencies would correspond to the lower end of the audible range ( 50 - $100 \mathrm{~Hz}$ ). Effective attenuation could be achieved at higher frequencies, however, by making use of multitube nozzles. Dean and Tester (1975) have already exploited this silencing mechanism by means of a bias air flow through an acoustic wall liner, an expedient originally proposed for this purpose by Barthel (1958).

The author gratefully acknowledges the benefit of discussions of the material of this report with Dr. -Ing. D. Bechert of Deutsche Forschungs- und Versuchsanstalt für Luft- und Raumfahrt, Berlin, and Dr. R.M. Munt of the University of Dundee. 


\section{APPENDIX}

\section{Compact Green's Function for an Axi-Symmetric Nozzle}

In the absence of a mean flow Ffowcs Williams and Howe (1975) have given the following expression for the compact approximation to the Green's function for an axi-symmetric nozzle of the type shown in Fig. l:

$$
\left.G(\underset{\sim}{x} \underset{\sim}{y}, t, \tau)=\frac{I}{4 \pi|\underset{\sim}{x}|} \delta \int_{t}-\tau-\frac{\left(|\underset{\sim}{x}-\underset{\sim}{K}(y)|-\frac{C_{0}}{C_{1}} F_{A}(\underset{\sim}{y})\right)}{c_{0}}\right\}(A . I)
$$

This is suitable for treating aerodynamic noise problems in which the characteristic wavelength of the sound is large compared with the nozzle radius. The observer is located in free space at the far field point $x, c_{0}$ is the sound speed in the ambient medium and $c_{1}$ that upstream of the nozzle exit.

The functions $\mathrm{F}_{\mathrm{A}}(\mathrm{y}), \underset{\sim}{\mathrm{N}}(\mathrm{y})$ are harmonic, and satisfy the normal velocity condition $\underset{\sim}{\tilde{n}} \cdot \tilde{\nabla}\left(F_{A}, \underset{\sim}{K}\right)=0$ on the walls of the rigid nozzle, $\underset{\sim}{n}$ being the unit normal. In particular, $F_{A}(y)$ is the poitential of an axi-symmetric incompressible nozz̃le flow, and, taking the coordinate origin in the center of the nozzle exit plane, as in the main text, is normalized such that:

(i) for $|\underset{\sim}{y}|>>a$ in free space

$$
F_{A}(\underset{\sim}{y})=-\frac{A}{4 \pi|\underset{\sim}{y}|} \quad ;
$$

(ii) within the nozzle in the vicinity of the point $N$ of Fig. 1 ,

$$
F_{A}(\underline{y}) \simeq \frac{A}{A}\left(Y_{1}-\ell_{0}\right)
$$

$\ell=0.6133 a$ being the "end-correction" of a semi-infinite circular cylindrical pipe (Noble, 1958, page 138); 
(iii) upstream of the nozzle contraction

$$
F_{A}(\underset{\sim}{y}) \simeq y_{1}-\lambda,
$$

where $\lambda$ is the effective, geometric nozzle end correction given approximately by

$$
\lambda=\frac{A}{\bar{A}}\left\{\ell_{0}+\left(\Delta+\frac{L}{2}\right)\left(\frac{A-A}{A}\right)\right\}
$$

$\Delta$ being the length of the neck of the nozzle, and $L$ the axial distance over which the contraction occurs (Rayleigh 1945, Sec. 308).

The vector valued function $\underset{\sim}{\mathrm{K}}(\underset{\sim}{\mathrm{y}})$ has the following properties:

(i) for $|\underset{\sim}{y}| \gg$ a in free space

$$
\underset{\sim}{\mathrm{K}}(\underset{\sim}{\mathrm{y}}) \simeq \underset{\sim}{\mathrm{y}} \quad ;
$$

(ii) for $\left|y_{1}\right|>$ a in the nozzle

$$
\underset{\sim}{K}(\underset{\sim}{\mathrm{y}}) \simeq \text { constant }
$$

(iii) in the vicinity of the nozzle exit

$$
\underset{\sim}{\mathrm{K}}(\underset{\sim}{\mathrm{y}})=\left(\mathrm{y}_{1}-\frac{\mathrm{A}}{\bar{A}} \mathrm{~F}_{\mathrm{A}}(\underset{\sim}{\mathrm{y}}), \mathrm{F}_{\mathrm{B}}(\underset{\sim}{\mathrm{y}}), \mathrm{F}_{\mathrm{C}}(\underset{\sim}{\mathrm{y}})\right) ;
$$

the precise forms of the potential functions $F_{B}, F_{C}$ are not required in applications to axi-symmetric source distributions.

In the presence of a low Mach number mean flow from the nozzle, the representation (A.I) of the Green's function must be modified to read:

$$
G(\underset{\sim}{x}, \underset{\sim}{y}, t, \tau)=\frac{1}{4 \pi|x|} \delta\left\{t-\tau-\frac{\left(|\underset{\sim}{x}-\underset{\sim}{\operatorname{K}}(\underset{\sim}{y})|-\left(\frac{c_{0}}{c_{1}}\right) \frac{F_{A}(\underset{\sim}{)})}{(1+M}\right)}{c_{0}}\right\}
$$


In this expression the additional factor $(1+M)$, where $\mathrm{M}=\mathrm{U} / \mathrm{c}$, accounts for the convection of the "imploding" wave by the mean flow upstream of the nozzle contraction.

\section{Incompressible Pulsatile Nozzle Flow}

The potential $\Psi(x) e^{-i \omega t} \equiv\left[F_{A}(x)+F_{J}(x)\right] e^{-i \omega t}$ which describes incompressible pulsatile flow in the downstream portion of the nozzle in the presence of a mean flow may be estimated from the corresponding solution for a semi-infinite, circular cylindrical duct. In the case in which the shear layer of the exterior jet flow $1 \mathrm{~s}$ modeled by a linearly disturbed vortex sheet, the Wiener-Hopf procedure described by Munt (1977) for the compressible problem yields for the solution in which the Kutta condition is imposed:

$\Psi \underset{\sim}{(x)}=\lim _{\varepsilon \rightarrow+0} \frac{A}{A}\left\{-\frac{e}{2 \varepsilon}+\frac{a}{2 \varepsilon}^{-\varepsilon \pi} \int_{-\infty}^{\infty} \frac{\left(\omega-i \varepsilon U_{J}\right) F(k, r) k_{+}(i \varepsilon) k_{-}(k)}{|k|\left(\omega-U_{J} k\right) Z_{+}(i \varepsilon) Z_{-}(k)} e^{i k x_{1}} d k\right\}$ (A. 4)

where

$$
\begin{aligned}
F(k, r) & =I_{0}(|k| r) / I_{1}(|k| a) \quad(r<a) \\
& =-\omega K_{0}(|k| r) /\left(\omega-U_{J} k\right) K_{1}(|k| a) \quad(r>a)
\end{aligned}
$$

and the first term in the brace brackets of (A.4) is omitted when $r>a$. Here and elsewhere $|k|=\sqrt{k^{2}+\varepsilon^{2}}$, and $I_{n}, K_{n}$
are modified Bessel Functions of order $n$ (Abramowitz and Stegun, 1964, p. 374).

The various quantities appearing in these formulae are defined as follows. A function $f(k)$ which is regular and non-zero on the real $k$-axis defines functions $f_{ \pm}(k)$ respectively regular and non-zero in Im $k \geq 0$ by means of

$$
f_{ \pm}(k)=\exp \left\{ \pm \frac{1}{2 \pi i} \int_{-\infty}^{\infty} \frac{\ln f(\xi)}{\xi-k} \mathrm{~d} \xi\right\}
$$


provided that the integral exists in an appropriate sense (Noble, 1958, page 13).

The functions $K, Z$ are given by

$$
\begin{aligned}
K(k) & =2 I_{1}(|k| a) K_{1}(|k| a) \\
Z(k) & \equiv z\left(\frac{U_{J} k}{\omega}, k a, \frac{\rho_{0}}{\rho_{1}}\right) \\
& =|k| a\left\{\frac{\rho_{0}}{\rho_{1}} \cdot \frac{I_{1}(|k| a) K_{0}(|k| a)}{\left(1-\frac{U_{J} k}{\omega}\right)^{2}}+I_{0}(|k| a) K_{1}(|k| a)\right\}
\end{aligned}
$$

As $\mathrm{x}_{1} \rightarrow-\infty$ within the circular cylindrical duct the principal contribution to the integral in (A.4) is from a simple pole at $k=-i \varepsilon$. This yields the approximate form of $\Psi$ close to the point $N$ of the nozzle of Fig. I, viz:

$$
\Psi \simeq \lim _{\varepsilon \rightarrow+0} A \frac{A}{A}\left\{-\frac{e^{-\varepsilon X_{i}}}{2 \varepsilon}+\left(\frac{\omega-i \varepsilon U_{J}}{\omega+i \varepsilon U_{J}}\right) \frac{K_{+}(i \varepsilon) K_{-}(-i \varepsilon)}{Z_{+}(i \varepsilon) Z_{-}(-i \varepsilon)} \frac{e^{\varepsilon x_{i}}}{2 \varepsilon}\right\}
$$

Now as $\varepsilon \rightarrow 0$

$$
K_{+}(i \varepsilon) K_{-}(-i \varepsilon) \simeq I-2 \varepsilon l_{0},
$$

where $l_{0}=0.6133 a$ is the end correction of a circular cylindrical pipe (Noble 1958, p. 138).

The solution (A.4) will satisfy the causality condition, i.e., the condition that the fluctuations in the exterior flow are a consequence of the pulsating nozzle flow, provided that it is regular in an upper complex w-plane (Lighthill, $1960)$. For $\operatorname{Im} \omega \sim+\infty$ the dispersion function $Z(k) \rightarrow I / 2$ as $k \rightarrow \pm \infty$ on the real axis and

$$
z_{+}(i \varepsilon) z_{-}(-i \varepsilon)=\exp \left\{\frac{\varepsilon}{\pi} \int_{-\infty}^{\infty} \frac{2 n z(k) \cdot d k}{k^{2}+\varepsilon^{2}}\right\}
$$


The result for real $w$ is obtained by analytic continuation, and as $w$ approaches the positive real axis, say, the zero $k=k_{1}$ of the dispersion function $Z(k)$ which corresponds to the instability mode of the semi-infinite jet crosses the real $k$-axis from the first quadrant into the fourth quadrant. Deforming the contour in (A.IO) to take account of this, and comparing the result with the integral along the real k-axis, we find that for real, positive $\omega$

$$
z_{+}(i \varepsilon) z_{-}(-i \varepsilon) \simeq \exp \left\{\frac{\varepsilon}{\pi} \int_{-\infty}^{\infty} \frac{2 n z(k) d k}{k^{2}}+\frac{2 i \varepsilon}{k_{I}}\right\}
$$

The integral here is split into real and imaginary parts by noting that as $\operatorname{Im} \omega \rightarrow+0$ the argument of $z(k)$ decreases discontinuously by $2 \pi$ as $k$ increases through $k=\omega / U_{J}$, and in this way we find that for small $\varepsilon$

$$
z_{+}(i \varepsilon) z_{-}(-i \varepsilon) \simeq \exp \left\{\frac{\varepsilon}{\pi} \int_{-\infty}^{\infty} \frac{2 n|z(k)| d k}{k^{2}}-\frac{2 i \varepsilon U_{J}}{\omega}+\frac{2 i \varepsilon}{k_{I}}\right\}
$$

Substitution of (A.9), (A.12) into (A.8) gives the limiting value

$$
\Psi=\frac{A}{A}\left\{x_{1}-\ell_{0}-\frac{U_{J}}{\omega}(\zeta-\mu)-i \frac{U_{J}}{\omega} \nu\right\},
$$

where $\zeta, \mu, v$ are defined in $(3.32),(3.34)$. Reference to the defining properties of $\mathrm{F}_{\mathrm{A}}(\underset{\sim}{\mathrm{x}})$ given above then shows that

$$
\mathrm{F}_{\mathrm{J}} \underset{\sim}{(\mathbf{x})} \rightarrow-\frac{A}{\mathrm{~A}} \frac{\mathrm{U}_{\mathrm{J}}}{\omega}((\zeta-\mu)+i \nu),
$$

upstream of the nozzle contraction, which immediately leads to $(3.31 a, b)$. 
The Case of a Finite Width Shear Layer

To solve (3.36) first set in the linearized approximation

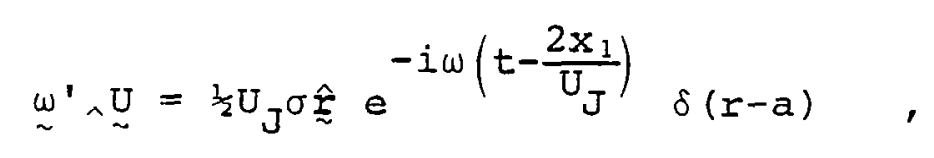

and solve $(3.36)$ by the Wiener-Hopf procedure to give

$$
B_{J}=\lim _{\varepsilon \rightarrow+0} \frac{a \omega \sigma}{4} \frac{\pi}{i} k_{+}\left(\frac{2 \omega}{U_{J}}\right) \int_{-\infty}^{\infty} \frac{(k-i \varepsilon) k_{-}(k) I_{\ell}(|k| r)}{|k| I_{1}(|k| a)\left(k-2 \omega / U_{J}\right)} e^{i k x_{1}} d k
$$

for $r \leqq a$.

Write $B_{A}=\beta B_{I} F_{A}(\underset{\sim}{x})$. The circulation density $\sigma$ of the shed vorticity is chosen to ensure that $B_{A}+B_{J}$ satisfies the Kutta condition at the nozzle lip. An integral expression for $B_{A}$ is obtained by setting $U_{J} \equiv 0$ in (A.4), and by considering the behavior of the integrands in (A.4), (A.16) as $k \rightarrow \infty$ it follows in the usual way (Jones 1972) that

$$
\sigma=\frac{-i A \beta B_{I}}{\omega A K_{+}\left(\frac{2 \omega}{U_{J}}\right)}
$$

This may be used in (A.6) to determine the upstream limiting form of $B_{J}$ and thence to give (3.37). 


\section{REFERENCES}

Abramowitz, M. and Stegun, I.A. (1964), Handbook of Mathematical Functions, Nat. Bur. Standards, Appl. Math. Series, No. 55 .

Barthel, Von F. (1958) "Untersuchungen uber nichtlineare Helmholtzresonatoren," Fequenz, 12, 1-11.

Bechert, D. and Pfizenmaier, E. (1975a), "On the amplification of broad band jet noise by a pure tone excitation," J. Sound Vib., $43,581-587$.

Bechert, D. and Pfizenmaier, E. (1975b), "Optical compensation measurements on the unsteady exit condition at a nozzle discharge edge," J. Fluid Mech., 71, 123-144.

Bechert, D., Michel, U. and Pfizenmaier, E. (1977), "Experiments on the transmission of sound through jets," AIAA Paper 77-1278.

Blokhintsev, D.I. (1946), "Acoustics of a nonhomogeneous moving medium," N.A.C.A. Tech. Memo. No. 1399.

Brown, G.B. (1935), "On vortex motion in gaseous jets and the origin of their sensitivity to sound," Proc. Phys. Soc., 47,703 .

Crighton, D.G. (1972), "The excess noise field of subsonic jets," U.K. Aero. Res. Counc. 33714 N. 781.

Crow, S.C. (1972), "Acoustic gain of a turbulent jet," Meeting Div. Fluid. Dyn. Am. Phys. Soc. Univ. Colorado, paper IEG.

Davies, P.O.A.L., Fisher, M.J. and Barrat, M.J. (1963), "The characteristics of the turbulence in the mixing region of a round jet," J. Fluid Mech. 15, 337-367.

Dean, P.D. and Tester, B.J. (1975), "Duct Wall impedance control as an advanced concept for acoustic suppression," NASA CR-134998.

Ffowcs Williams, J.E. and Howe, M.S. (1975), "The generation of sound by density inhomogeneities in low Mach number nozzle flows," J. Fluid Mech. 70, 605-622. 
Freymuth, P. (1966), "On transition in a separated laminar. boundary laoyer," J. Fluid Mech., 25, 683-704.

Gerend, R.P., Kumasaka, H.P. and Roundhill, J.P. (1973), "Core engine noise," AIAA paper No: 73-1027.

Howe, M.S. (1975), "Contributions to the theory of aerodynamic sound, with application to excess jet noise and the theory of the flute," J. Fluid Mech., 71, 625-673.

Jones, D.S. (1972), "Aerodynamic sound due to a source near a half-plane," J. Inst. Math. Applic. 9, 114-122.

Landau, L.D. and Lifshitz, E.M. (1959), Fluid Mechanics, Permagon-Oxford.

Liepmann, H.W. and Roshko, A. (1957), Elements of Gasdynamics, Wiley-New York.

Lighthill, M.J. (1952), "On sound generated aerodynamically. I. General theory," Proc. Roy. Soc. Lond. A211, 564-587.

Lighthill, M.J. (1960), "Studies on magneto-hydrodynamic waves and other anisotropic wave motions," Phil. Trans. Roy. Soc. Lond., A252, 397-430.

Mechel, F.P., Mertens, P.A. and Schiltz, W.M. (1967), "Interaction between air flow and airborne sound in a duct," Aero. Med. Res. Lab.-TR-67-120(II), Aero. Med. Div. Air Force Systems Comm., Wright-Patterson Air Force Base, Ohio.

Moore, C.J. (1977), "The role of shear layer instability waves in jet exhaust noise," J. Fluid Mech. 80, 321-367.

Munt, R.M. (1977), "The interaction of sound with a subsonic jet issuing from a semi-infinite cylindrical pipe," J. Fluid Mech. 83, 609-640.

Noble, B. (1958), Methods based on the Wiener-Hopf technique., Pergamon-Oxford.

Pinker, R.A. and Bryce, W.D. (1976), "The radiation of plane wave duct noise from a jet exhaust, statically and in flight," U.K. Nat. Gas. Turbine Est. Note No. NT-1024.

Rayleigh, Lord (1945), The theory of sound, Dover, New York. 
Saffman, P.G. (1975), "On the formation of vortex rings," Stud. Appl. Math. 54, 261-268.

Savkar, S.D. (1945), "Radiation of cylindrical duct acoustic modes with flow mismatch," J. Sound Vib. 42, 363-386.

Stratton, J.A. (194I), Electromagnetic Theory, McGraw HilI, New York. 


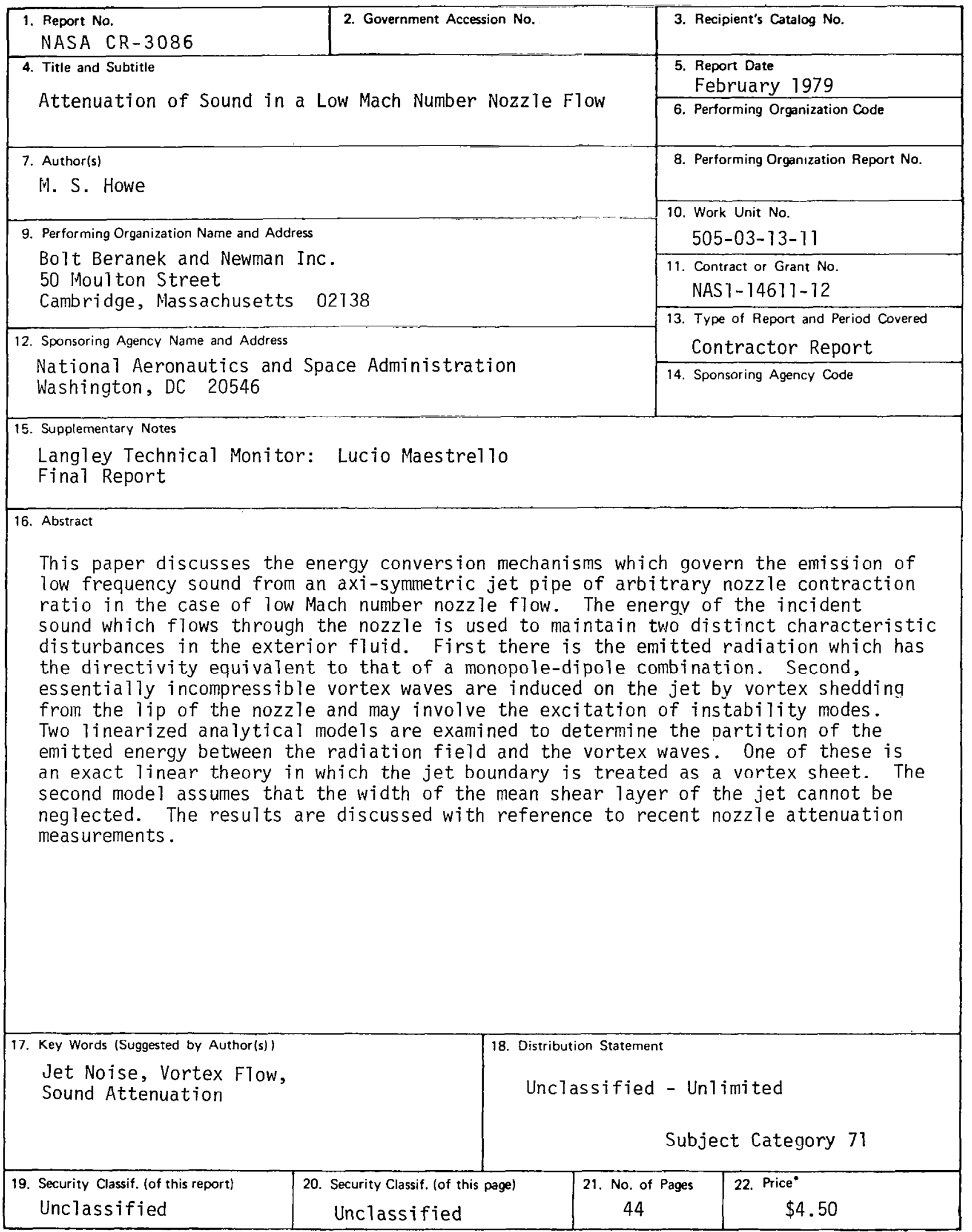

*For sale by the National Technical Information Service. Springfield, Virgınia 22161 